complexes were enriched in MDVs when the outer-membrane-localized deubiquitylase USP30 was depleted ${ }^{2}$. Loss of USP30 affects the stability of the TOM complex and impairs deubiquitylation of mitochondrial precursor proteins at the TOM complex ${ }^{11}$. Altogether, MDVs promoted the degradation of $\beta$-barrel proteins and protein complexes, which represent highly stable folds that are difficult to extract from membranes.

The proteome of TOM-MDVs also provided new insights in the formation of these vesicles. The outer-membrane proteins mitochondrial Rho-GTPase 1 and 2 (MIRO1 and MIRO2) were present in TOM-MDVs, which was confirmed by super-resolution microscopy. The formation of TOMMDVs depended on both MIRO1/2 and microtubule dynamics, and the authors found thin TOMM20-eGFP-positive membrane protrusions forming at mitochondria (where eGFP denotes enhanced green fluorescent protein). Live-cell imaging revealed that these protrusions are the source of TOM-MDVs, which are later degraded in lysosomes. This finding indicated that MIRO1/2 promote the directed formation of protrusions, from which TOM-MDVs are pinched off. König et al. ${ }^{2}$ then studied how TOM-MDVs are separated from mitochondria. They found that TOM-MDVs were enriched in phosphatidic acid, and alteration of the phosphatidic acid content in TOM-MDVs revealed a critical role for this phospholipid in the formation of MDVs. Phosphatidic acid is known to cooperate with the large GTPase dynamin-related protein 1 (DRP1) to drive mitochondrial division ${ }^{12}$. Studies of DRP1 knockout cells showed that active DRP1 was required for MDV formation. Excitingly, DRP1 assembled into punctate structures at MDV formation sites close to the tip of mitochondrial protrusions as shown by live-cell imaging (Fig. 1). Thus, DRP1 and phosphatidic acid promote the fission of MDVs from mitochondria.

Altogether, the presented proteomic and lipidomic analyses of MDVs uncovered the central function of MDVs in mitochondrial protein quality control and revealed major steps in MDV formation at mitochondria. The observed role of MDVs in the removal of mitochondrial $\beta$-barrel proteins and protein complexes establishes MDVs as an important mechanism in the surveillance of the mitochondrial proteome. Future studies will have to address several questions about MDV formation. How are defective $\beta$-barrel proteins and protein complexes recognized and delivered to form MDVs? What are the signals that mediate delivery of these cargos to MDVs? What are the functions of the different types of MDV? How is the formation of MDVs regulated? Analyses of cargo selection and its transfer to MDVs will help us to understand how mitochondria communicate with other cell organelles.

Dominic Winter (D) and Thomas Becker (D) $₫$ Institute of Biochemistry and Molecular Biology, Faculty of Medicine, University of Bonn,

Bonn, Germany.

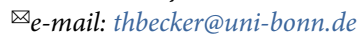

Published online: 6 December 2021 https://doi.org/10.1038/s41556-021-00801-y

References

1. Song, J., Herrmann, J. M. \& Becker, T. Nat. Rev. Mol. Cell Biol. 22 54-70 (2021).

2. König, T. et al. Nat. Cell Biol. https://doi.org/10.1038/s41556-02100798-4 (2021).

3. Soubannier, V. et al. Curr. Biol. 22, 135-141 (2012).

4. Neuspiel, M. et al. Curr. Biol. 18, 102-108 (2008).

5. McLelland, G. L. et al. EMBO J. 33, 282-295 (2014).

6. McLelland, G. L. et al. J. Cell Biol. 214, 275-291 (2016).

7. Suguira, A. et al. Nature 542, 251-254 (2017).

8. Matheoud, D. et al. Cell 166, 314-327 (2016).

9. Hughes, A. L. et al. eLife 5, el3943 (2016).

10. Schuler, M. H. et al. Mol. Cell 81, 3786-3802 (2021)

11. Phu, L. et al. Mol. Cell 77, 1107-1123 (2020).

12. Adachi, Y. et al. Mol. Cell 63, 1034-1043 (2016).

Competing interests

The authors declare no competing interests.

\title{
Profiling and promise of supermeres
}

\author{
Extracellular vesicles and particles have important roles in physiology and disease. Advances in isolation and \\ characterization technologies have enabled the identification of new particles. Supermeres are the newest addition \\ to the rapidly expanding repertoire of the cell secretome, and provide exciting opportunities for clinical translation.
}

\section{James W. Clancy, Alex C. Boomgarden and Crislyn D'Souza-Schorey}

$\mathrm{C}$ ells constantly release molecular material, much of which we now know can modulate physiological processes and be non-invasively accessed from biofluids to serve as molecular biomarkers. In particular, the past decade has seen a huge rise in research in extracellular vesicles because of their roles in intercellular communication and therapeutic potential as effective biomarkers in cancer and other diseases ${ }^{1}$. It is now appreciated that the term extracellular vesicle refers to the heterogeneous family of membrane-bound vesicles that are released by almost all cell types. More recently, further refinement of isolation techniques has led to the identification of new extracellular particles, termed exomeres ${ }^{2}$. These small, 35-nm nanoparticles lack an encompassing membrane but contain a unique signature of bioactive components, including protein, nucleic acid, lipid and N-glycosylation, which together suggest distinct biological functions. Amembranous nanoparticles with similar content and morphology could be isolated by ultracentrifugation of the supernatant after the isolation of small extracellular vesicles $^{3}$. In this issue of Nature Cell Biology, Zhang et al. ${ }^{4}$ present findings that arise from investigations of the remaining supernatant after exomere isolation, and report the identification of additional nanoparticles that they have called supermeres (supernatant of exomeres).

The identification of supermeres represents a crucial step in the ongoing pursuit to define the repertoire of extracellular vesicles and particles (EVPs) (Fig. 1). By additional ultracentrifugation after the isolation of exomere particles, this work purified nanoparticles that differed in 


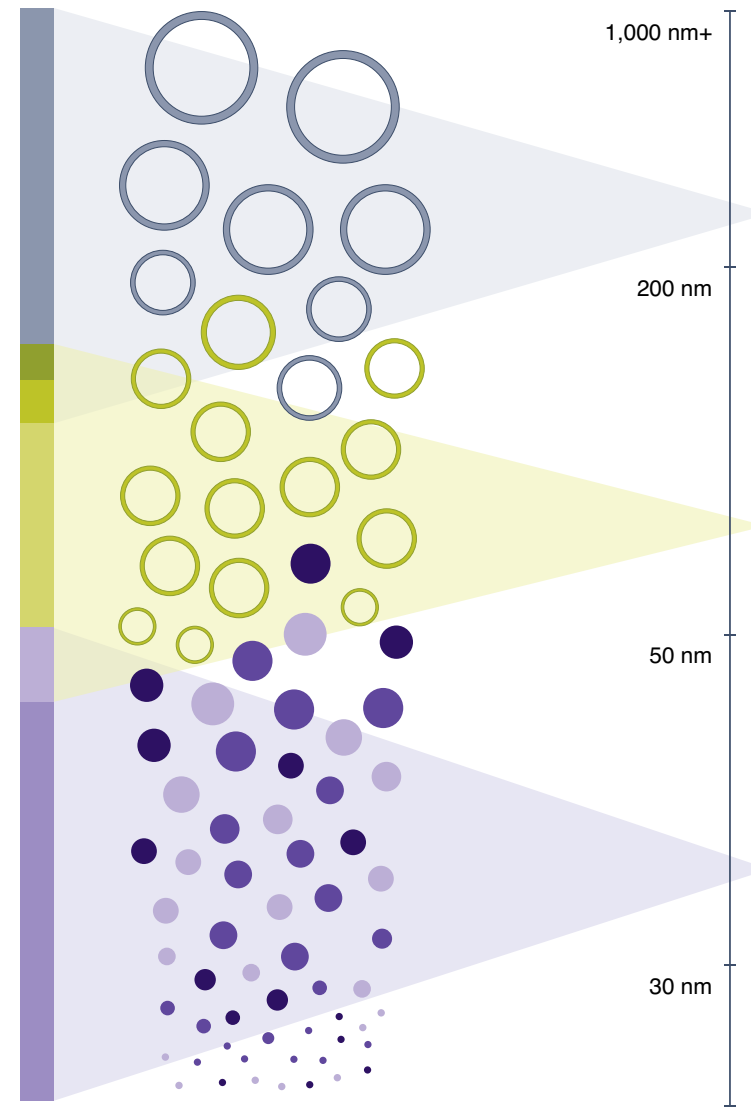

Large extracellular vesicles

Large oncosomes:

Size: $>1,000 \mathrm{~nm}$

Markers: CK18, GOT1, GAPDH,

glutaminase

Microvesicles:

Size: $200-1,000 \mathrm{~nm}$

Markers: ARF6, VAMP3, AnnexinA1

Small extracellular vesicles

Exosomes:

Size: $60-120 \mathrm{~nm}$

Markers: CD63, Syntenin-1, Alix, Rab27a

ARMM:

Size: 50-200 nm

Markers: ARRDC1, Tsg101

\begin{tabular}{|c|c|}
\hline \multicolumn{2}{|l|}{ Extracellular particles } \\
\hline $\begin{array}{l}\text { Exomere: } \\
\text { Size: <50 nm } \\
\text { Markers: Hsp90- } \beta \text {, } \\
\text { ENO1, GANAB }\end{array}$ & $\begin{array}{l}\text { HDL: } \\
\text { Size: 8-12 nm } \\
\text { Markers: ApoA1 }\end{array}$ \\
\hline \multirow[t]{2}{*}{$\begin{array}{l}\text { Supermere: } \\
\text { Size: <50 nm } \\
\text { Markers: TGF- } \beta \text { I, } \\
\text { AGO2, ACE2, PCSK9 }\end{array}$} & $\begin{array}{l}\text { LDL: } \\
\text { Size: 18-22 nm } \\
\text { Markers: ApoB100 }\end{array}$ \\
\hline & $\begin{array}{l}\text { VLDL: } \\
\text { Size: } 35-100 \mathrm{~nm} \\
\text { Markers: ApoB100 }\end{array}$ \\
\hline
\end{tabular}

Fig. 1 | Extracellular vesicles and particles. Large extracellular vesicles (L-EVs) include microvesicles and oncosomes and from by the outward budding of the plasma membrane. Small extracellular vesicles (S-EVs) are derived from multivesicular bodies as well as the cell surface. Larger S-EVs and smaller L-EVs overlap in size. Extracellular particles are complexes of proteins and nucleic acids but not membrane-enclosed. The mechanisms of extracellular particle biogenesis are unknown. Within each of these groups, several subpopulations are likely to exist. HDL, high-density lipoprotein; LDL, low-density lipoprotein; VLDL, very-low-density lipoprotein.

morphology, cellular uptake, biodistribution in vivo and associated bioactive

components. Zhang and colleagues ${ }^{4}$ have conducted a thorough analysis of supermere components, and show that this nanoparticle fraction is enriched in disease-related proteins that range from APP and APLP2 (Alzheimer's disease); and ACE and ACE2 (cardiovascular disease and COVID-19); to $\alpha$-enolase and glypican-1 (cancer) (Fig. 2). Similarly, sequencing analysis highlights the presence of numerous RNA species, including several microRNAs (miRNAs), together with miRNA-processing proteins such as AGO2. The identification of this rich plethora of bioactive molecules with distinct uptake kinetics raises interesting questions about the function of supermeres, and heightens interest in the potential of these particles as biomarkers for diseases.

Mounting evidence suggests that the functional capabilities of extracellular vesicles stems largely from their ability to transfer cargo from shedding cells and their ability to elicit phenotypic changes in recipient cells. These alterations in recipient cells can contribute to the creation of a premetastatic niche, promote neurodegeneration, facilitate the repair of cardiac cells, or ameliorate the immunosuppressive tumour microenvironment (TME) ${ }^{5,6}$. Initial experimentation using supermeres revealed potent effects on liver metabolism, likely arising from the enrichment of proteins that are involved in metabolic processes. Several cargo-trafficking pathways have now been identified that further the understanding of individual populations of extracellular vesicles ${ }^{7}$. The mechanisms involved in supermere biogenesis remain to be determined, and whether these mechanisms may be exploited to modulate the tumour microenvironment, as has been shown for exosomes ${ }^{8}$, will require further investigation.

Despite cancer being a spatially and temporally dynamic disease, the clinical evaluation of its molecular profile is routinely assessed by evaluation of surgically resected tissue. Analysis of these samples, although initially providing essential insights, is imperfect owing to inherent limitations, including selection bias and providing only a single snapshot in time. In comparison to tissue-based biopsies, liquid biopsy is less invasive, and access to disease-related molecules from the peripheral blood (or other bodily fluids) of patients has the potential to provide real-time information about disease diagnosis, classification, status and longitudinal monitoring of treatment responses. Although much of the initial work to investigate biomarkers in EVPs focused on microvesicles and exosomes, it was recently reported that the plasma EVP proteome could be used to detect cancer with high sensitivity and specificity, and could distinguish among patient cancer types $^{9,10}$. The detection of supermeres in human plasma samples places them in line with microvesicles, exosomes and exomeres as targets for the development of liquid biopsies. Zhang and colleagues ${ }^{4}$ have taken the initial steps to examine the functionality of supermeres, and identified TGF- $\beta 1$ as one of the most abundant proteins in supermeres isolated from the plasma of patients with colorectal cancer, which further confirms that there is great promise in the development of EVP-based biomarkers.

Deepening current understanding of EVP structures and identification of new EVP types has relied on the optimization of established protocols and, more importantly, the development of techniques for their isolation and separation. So far, the predominant method used to fractionate and purify EVPs from conditioned cell culture medium or biofluid specimens has been serial centrifugation $^{11}$. Although this method has proven effective in its ability to generate bulk vesicle populations for downstream analysis, emerging reports have made it increasingly apparent that these sedimented fractions are enriched for certain classes of EVPs and probably contain a mixture of EVPs with other uncharacterized nanostructures and confounding serum proteins $s^{6}$. This has motivated researchers to develop and optimize higher resolution iodixanol or sucrose gradients to refine the fractions obtained by serial centrifugation protocols, enabling the separation of nanoparticles and polymers and increased fraction purity ${ }^{12}$. Although this has facilitated the further characterization of subclasses of extracellular vesicles - particularly microvesicles and exosomes - the application of this method to specifically isolate and characterize smaller, non-vesicular extracellular particles 


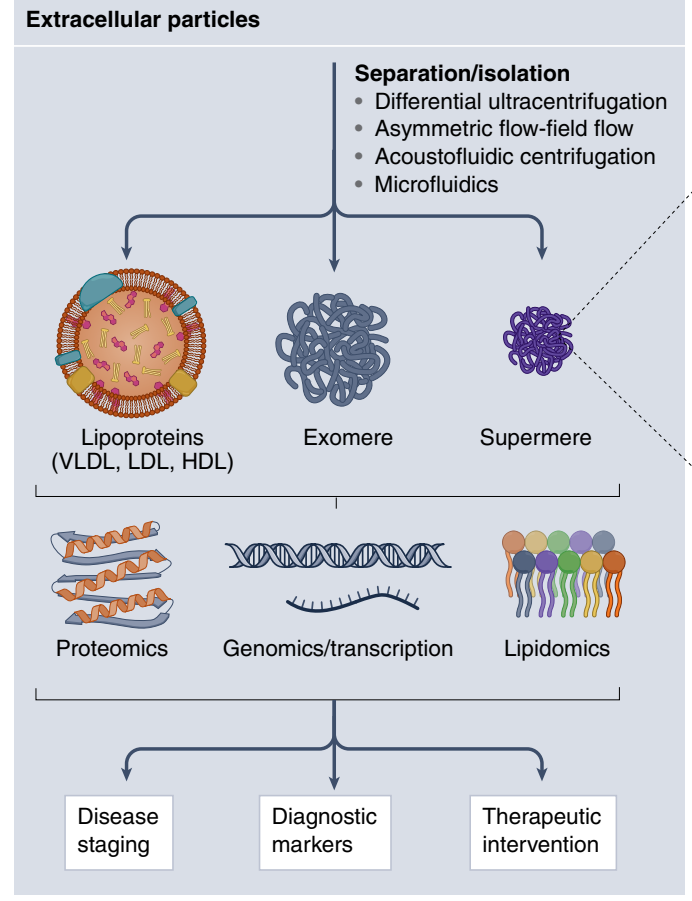

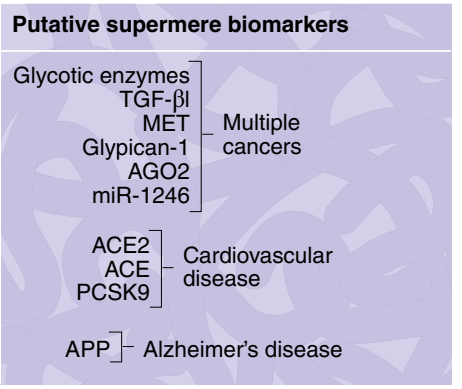

Fig. 2 | Extracellular particle isolation strategies and clinical potential. Although asymmetric flow field-flow fractionation (AF4) and serial ultracentrifugation have been used effectively to isolate extracellular particles, acoustofluidic centrifugation and microfluidic platforms are additional promising platforms. Extracellular particles can be useful for disease detection, disease staging and as reservoirs of potential therapeutic targets. A comprehensive proteomic analysis of supermeres by Zhang et al. ${ }^{4}$ identifies putative biomarkers for various diseases. VLDL, very-low-density lipoprotein. particle' encompasses all cell-secreted nucleic acid-protein complexes, and whether this would include previously described complexes such as extracellular chromatimeres ${ }^{15}$. Research is required to address these possibilities and distinguish these newly described EPs from other components of the cellular secretome. Functional and proteomic comparisons conducted as part of the research proposing supermeres as a novel extracellular particle underscores this possibility, as similar pathophysiological effects were seen in the livers of mice subjected to systemic treatment with either exomeres or supermeres. Interestingly, signalling differences emerged in the livers of mice treated with exomeres versus those treated with supermeres. Similarly, the supermeres isolated from a cell model of colorectal cancer shared more identified targets in common with exomeres, than were distinct from them, although the bulk of the proteins identified within isolated exomeres were common to both exomeres and supermeres. The desire to translate EVP biology from the bench (or centrifuge) to the bedside relies on properly defining, describing and attributing content and biological action to the specific particle type. The discovery of supermeres represents a large and important step in this direction, replete with exciting opportunities for adaptation and clinical translation.

James W. Clancy, Alex C. Boomgarden and Crislyn D'Souza-Schorey (D) $ه$

Department of Biological Sciences, University of

Notre Dame, Notre Dame, IN, USA.

$凶_{e-m a i l: c d s o u z a s @ n d . e d u}$

Published online: 9 December 2021

https://doi.org/10.1038/s41556-021-00808-5 considerable strides made in the field although their application in clinical settings is still reliant on improving the cost of laboratory instrumentation, scale of sample preparation and speed.

Perhaps the most important challenge facing the field of extracellular vesicle or EVP biology, and one that weighs heavily on the prospect of future clinical relevance of EVP-based biotechnology, is a comprehensive understanding of EVP heterogeneity, both between and within EVP classes. The most well-characterized EVP intraclass heterogeneity has emerged from EVP biologists, who have begun a painstaking classification of the distinct subtypes of particles broadly classified as exosomes $^{2,12}$. It remains to be seen how, or even if, similar categorization exists within a larger EVP class of nanoparticles, with exomeres and supermeres representing the first identified subtypes. It also remains unclear whether the term 'extracellular

\footnotetext{
References

1. van Niel, G., D’Angelo, G. \& Raposo, G. Nat. Rev. Mol. Cell Biol. 19, 213-228 (2018).

2. Zhang, H. et al. Nat. Cell Biol. 20, 332-343 (2018).

3. Zhang, Q. et al. Cell Rep. 27, 940-954 (2019).

4. Zhang, Q. et al. Nat. Cell Biol. https://doi.org/10.1038/s41556021-00805-8 (2021).

5. O'Brien, K., Breyne, K., Ughetto, S., Laurent, L. C. \& Breakefield, X. O. Nat. Rev. Mol. Cell Biol. 21, 585-606 (2020).

6. Sheehan, C. \& D'Souza-Schorey, C. J. Cell Sci. 132, jcs 235085 (2019).

7. Clancy, J. W., Schmidtmann, M. \& D'Souza-Schorey, C. FASEB Bioadv. 3, 399-406 (2021).

8. Wang, S. et al. Sci. Transl. Med. 13, eabb6981 (2021).

9. Hoshino, A. et al. Cell 182, 1044-1061 (2020).

10. Mendt, M. et al. JCI Insight 3, 3e99263 (2018).

11. Thery, C., Amigorena, S., Raposo, G. \& Clayton, A. Curr. Protoc. Cell Biol. Ch. 3, Unit 3.22 (2006).

2. Jeppesen, D. K. et al. Cell 177, 428-445 (2019).

13. Cheng, S. et al. Lab Chip 21, 3219-3243 (2021).

14. Gu, Y. et al. Sci. Adv. https://doi.org/10.1126/sciadv.abc0467 (2021).

15. Choi, D. et al. ACS Nano 13, 10499-10511 (2019).
}

Competing interests

The authors declare no competing interests. 